\title{
NUMERICAL ANALYSIS OF THE AIR-FUEL MIXTURE IN INDIRECT AND DIRECT INJECTION OF FOUR-STROKE ENGINES
}

\author{
G. G. Narcizo, \\ and D. A. Miranda \\ Universidade da Região de Joinville \\ UNIVILLE \\ Curso de Engenharia Mecânica \\ Rua Norberto Eduardo Weihermann, 230 \\ São Bento do Sul, Santa Catarina, Brasil \\ gedinarcizo@gmail.com \\ diegoalves_klx@hotmail.com \\ ABSTRACT \\ The quality of the air-fuel mixture in internal combustion engines directly \\ affects the combustion efficiency, therefore a good design of the \\ combustion chamber combined with the correct fuel injection system, can \\ provide a better use of this mixture and increase the efficiency of the \\ engine. Considering these aspects, this scholarly work presents a \\ comparative study of the indirect injection system and direct fuel injection, \\ analyzing the way the mixture behaves in these two conditions. For this, the \\ Ansys Fluent simulation software was used, in which were applied \\ computational fluid dynamics simulations of the air-fuel mixture. The \\ objective of this scholarly work is to contribute to the development of the \\ injection systems, enabling the improvement of new studies and \\ developments of new nozzle models can be performed. \\ Received: March 05, 2019 \\ Revised: April 15, 2019 \\ Accepted: July 03, 2019 \\ Keywords: numerical simulation; combustion efficiency; formation of air- \\ fuel mixture
}

\section{NOMENCLATURE}

K Thermal Conductivity, W/m.K

$\mathrm{k} \quad$ Turbulent kinetic energy, $\mathrm{m}^{2} / \mathrm{s}^{2}$

$S_{\mathrm{i}} \quad$ Reynolds tensor, $\mathrm{Pa}$

$S_{\mathrm{Mi}} \quad$ Source term of the momentum, $\mathrm{Pa}$

$\mathrm{T}$ Temperature, $\mathrm{K}$

$\mathrm{t}$ time, $\mathrm{s}$

u Velocity, $\mathrm{m} / \mathrm{s}$

$\mathrm{U}$ Mean velocity, $\mathrm{m} / \mathrm{s}$

$x \quad$ Coordinates, $\mathrm{m}$

$p$ Pressure, $\mathrm{Pa}$

\section{Greek symbols}

\section{$\sigma \varepsilon \quad$ Closure constant, $\mathrm{Pa}$}

$\sigma_{\mathrm{k}} \quad$ Closing constant, $\mathrm{Pa}$

$\rho \quad$ Density $\mathrm{kg} / \mathrm{m}^{3}$

$\vec{\nabla} \quad$ Vector operator gradient

$\varepsilon \quad$ dissipation rate, $\mathrm{m}^{2} / \mathrm{s}^{3}$

$\mu \quad$ Dynamic viscosity, Pa.s

$\mu_{\mathrm{t}} \quad$ Turbulent dynamic viscosity, Pa.s

$\varphi \quad$ Energy dissipation, $\mathrm{J}$

\section{INTRODUCTION}

With the evolution of computers, the capabilities of numerical tools have been increased, which are used to study internal phenomena in the engines and other subjects, injection and mixing formation receive great attention (Miranda et al. 2018). This application allows sensitivity studies and definitions of components prior to prototype production need. The benefits include reducing time and cost of development.

As reported by Zhao et al., (appud., Guzzo 2012), many engineers have devoted their studies and efforts to the development of engines that provide, not only a reduction in fuel consumption, but also a great reduction of pollutant emissions, which is directly linked to the burning efficiency. Kasper (2003), explains that the performance of internal combustion engines and its pollutant emissions are governed by non-stationary fluid-dynamic processes. The importance of good understanding of these processes is indispensable to optimize engine characteristics, and computational fluid dynamics (CFD) techniques are capable to represent detailed information about this flow. Fortunately, the modeling and simulation of internal combustion engines has been increasingly common in the scientific environment, because the need to become aware of this process is a great challenge, and can bring numerous benefits, both for the consumer and for the environment, in terms of emissions.

A CFD work applied to internal combustion engines that had a great degree of importance was the work of Kim et al., (1999), where the objective was to investigate the transient air flow in cylinders with the characteristics of spraying the fuel by means direct injection. For this purpose, the KIVA-3V code was used as a fuel spraying model to simulate the stratification of a late injection mode. In order to better understand the flow and the intake of the 
mixture inside the cylinder, the authors used two base geometries of the piston, where one is flat and the other is irregular. It was concluded that for both simulations the behavior of the spray showed to be similar.

Another study that was accomplished in order to investigate the flow of the air-fuel mixture was presented by Milton et al., (2001), where the authors conducted an experimental study to analyze the mixture in an inlet valve of a combustion engine, along with numerical results obtained by means of simulation. It can be perceived that, the location of the injector nozzle, the opening of the valve and the inlet flow of the mixture, in this case being an indirect injection, have a great degree of relevance so that the fuel can be atomized and dispersed efficiently within the cylinder. The researchers reproduced the experimental data with simulation, where the FLUENT package was used. As the main conclusion, the researchers could perceive that the fluid-dynamic simulation was able to qualitatively reproduce the flow behavior inside the cylinder. In his study, Kim et al. (2008), argues the importance of the application of modeling and simulation in engines, because due to the complexity of the parameters to be analyzed, through the fluid-dynamic simulation it is possible to overcome several constraints, and this can provide researchers a better understanding of the flow, mixture formation and combustion process in the engines.

This study consists of applying modern computational fluid-dynamics techniques in the study of the flow of two fluids: atmospheric air and gasoline, into the combustion chamber of a fourstroke spark ignition engine. The combustion chamber in question is the HONDA UMK 435T engine, which is used in the Univille's Energy Efficiency Program - São Bento do Sul Campus. Research and comparison of two fuel injection methods will be carried out: indirect injection and direct injection, aiming to understand, compare and contribute to the development of the project and seek a better burning efficiency with reduction of pollutants. The problem approach will be twodimensional, by consuming less computational resource.

\section{METHODOLOGY}

In this chapter will be presented the methodology used to simulate the air-fuel mixture in situations of indirect injection and direct injection. The engine of interest, which was used as a source of studies is a four-stroke HONDA UMK 435T engine and powered with conventional gasoline.

\section{Domain}

It can be noticed in the Fig. 1 a cross-section view of the intake manifold, where the air-fuel mixture occurs - which will be the target geometry for the study of the indirect injection - and the combustion chamber - which will be the target geometry for the direct injection study - of the engine used in this scholarly work.

It is a three-dimensional design and it was developed in the SolidWorks CAD software. In order to be able to perform the simulations intended with this study, and due to low computational resource available, it was chosen to use of a two-dimensional representation software, which is exemplified in Fig. 2 , since a two-dimensional model does not require high processing capacities and excellent approximations can be achieved.
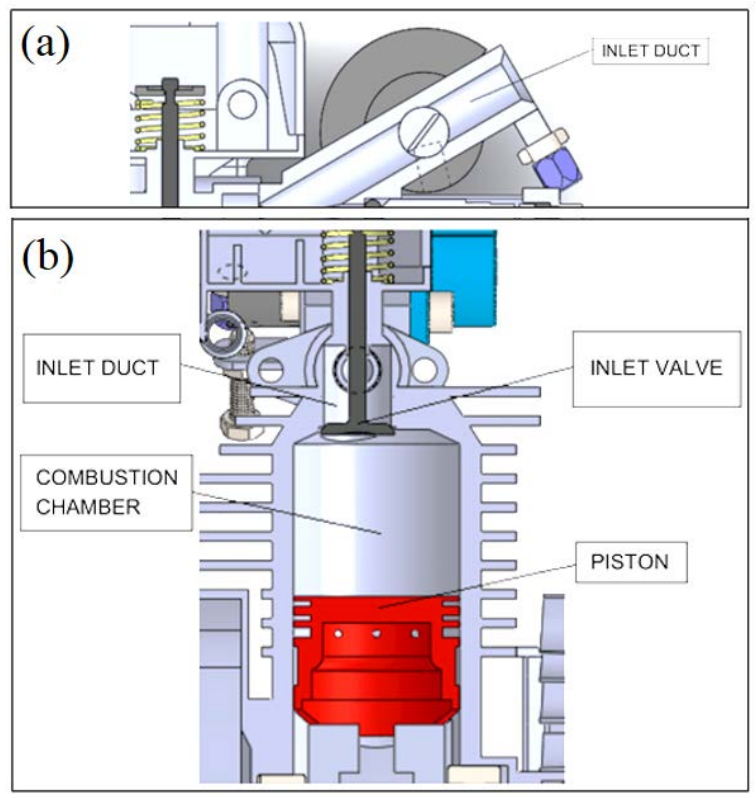

Figure 1. Cross-section view of HONDA UMK 435T engine. a) Intake manifold. b). Combustion chamber. Source: Adapted from Schreiner (2014).

The domains of interest for this study are the internal volume of the intake manifold and the engine cylinder, which are represented in Fig. 2. Figure 2 represents the computational domains used in this study. It is possible to perceive, for the case of indirect injection, presented in Fig. 2(a), an air intake duct and a representation of the injector nozzle.

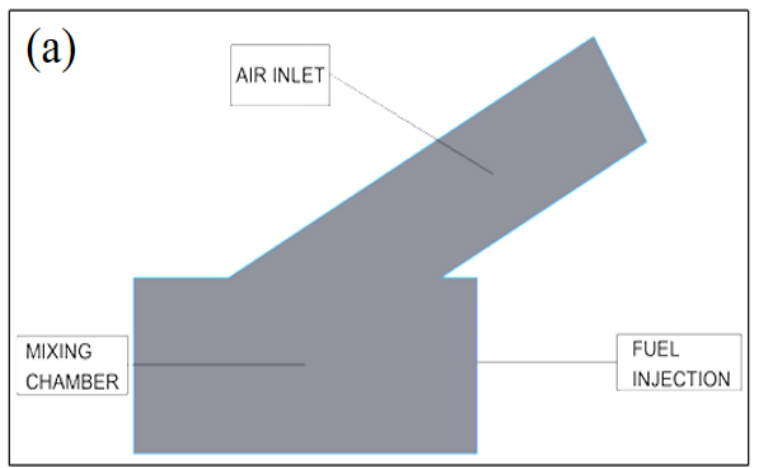




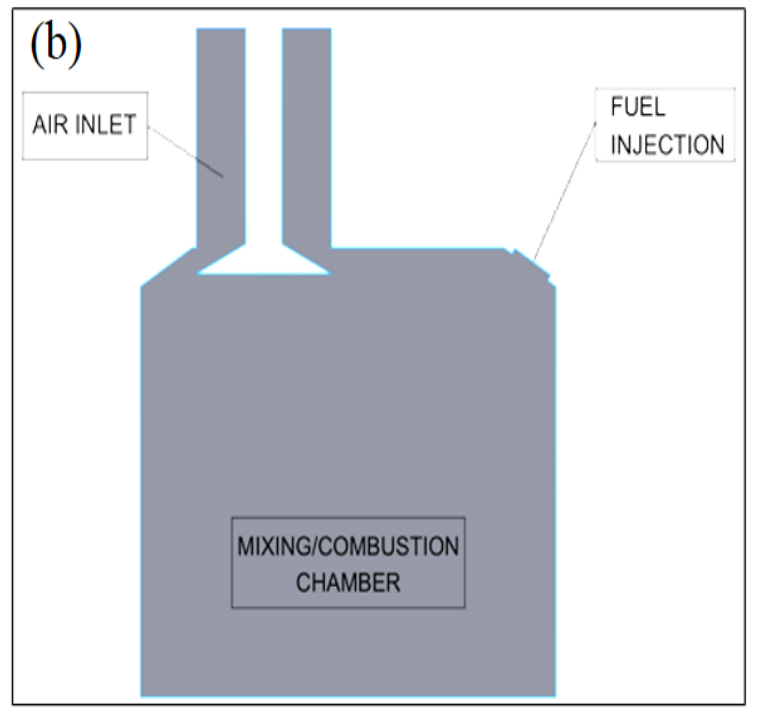

Figure 2. Schematic representation of computational domains. a) Indirect injection. b). Direct injection.

It is understood by the air-fuel mixing chamber, where atmospheric air flow and fuel injection occurs in a duct that precedes the inlet valve.

In Fig. 2(b), the combustion chamber is shown, where atmospheric air is admitted and the fuel injection is directly in it. Some considerations were necessary to design the two-dimensional domain, such considerations aimed to simplify the geometry to reduce the simulation time.

In the indirect injection model the mixture happens indirectly, that is, in a chamber that is located before the inlet valve. In the case of direct injection, it is perceived an air intake duct and the representation of the injector nozzle, whereby the fuel is injected directly into the combustion chamber. The domains in question were designed using SolidWorks CAD software. After the design of the domains, it was necessary to import them into the simulation software, which for this study was used the ANSYS Fluent.

\section{Government Equations}

In order to model any flow of a fluid, it is necessary to solve the equations of conservation of mass, amount of movement and energy.

$$
\frac{\partial \rho}{\partial t}+\vec{\nabla}(\rho u)=0
$$

According to Versteeg and Malalaskera (2007), the mass of the system remains constant and the result of the mass conservation Eq. (1) is the mass balance in a control volume. Where $\rho$ is the density of the fluid, $t$ is the time and $u$ is the velocity vector. The equation of the momentum for a control volume is determined by Newton's second law, which states that the sum of all external forces acting on the body is equal to the rate of change with time of the linear momentum, as Fox (2012).

$$
\rho \frac{D u}{D t}=-\frac{\partial p}{\partial x_{i}}+\vec{\nabla}(\mu \nabla u)+S_{M i}
$$

The Navier-Stokes Eq. (2) can be written in the most useful way for the development of the finite volume method, where $p$ is the pressure, $\mu$ is the dynamic viscosity and $S_{\mathrm{Mi}}$ is the source term of the momentum.

The CFD methodology seeks to report the average behavior of runoff fields in various physical phenomena. The energy equation is derived from the first law of thermodynamics, which states that the rate of energy change of a fluid particle is equal to the rate of addition of heat to the fluid particle plus the rate of work done on the particle.

$$
\rho \frac{D i}{D t}=-p \vec{\nabla} u+\operatorname{div}(k \nabla T)+\varphi+S_{i}
$$

After several rearrangements, the internal energy Eq. (3) is obtained using the Newtonian model for viscous stresses, as described by Versteeg and Malalasekera (2007) in his study. Where i is the internal energy per unit mass, $\mathrm{k}$ is the thermal conductivity, $\mathrm{T}$ is the temperature, $\varphi$ is the energy dissipation term and $\mathrm{S}_{\mathrm{i}}$ is the source term of the energy equation.

\section{Turbulence model k- $\varepsilon$}

$$
\begin{aligned}
\frac{\partial(k \rho)}{\partial t}+\vec{\nabla}(\rho k U) & =\vec{\nabla}\left(\frac{\mu_{t}}{\sigma_{k}} \nabla \varepsilon\right)+2 \mu_{t} \cdot S_{i j}-\rho \varepsilon \\
\frac{\partial(\rho \varepsilon)}{\partial t}+\vec{\nabla}(\rho \varepsilon U)= & \vec{\nabla}\left(\frac{\overrightarrow{\mu_{t}}}{\sigma_{\varepsilon}} \nabla \varepsilon\right) \\
& +C_{1 \varepsilon} \frac{\varepsilon}{k} 2 \mu_{t} \cdot S_{i j}-C_{2 \varepsilon} \rho \frac{\varepsilon^{2}}{k}
\end{aligned}
$$

The standard k- $\varepsilon$ model uses two Equations (4) and (5) to describe the turbulent kinetic energy $\mathrm{K}$ and the turbulent kinetic energy dissipation $\varepsilon$, respectively. The model describes how much kinetic energy is contained in the fluctuations, such consideration is observed by the letter k. However, the Greek letter $\varepsilon$ describes the rate of turbulent dissipation of the kinetic energy, $U$ is the mean velocity vector, $\mu_{\mathrm{t}}$ is the turbulent dynamic viscosity, $\sigma_{\mathrm{k}}$ is the closing constant of the k-omega model, $\mathrm{S}_{\mathrm{ij}}$ is the Reynolds tensor, $\sigma_{\varepsilon}$ is the closure constant of the model $\mathrm{k}-\varepsilon, \mathrm{C}_{1} \varepsilon$ and $\mathrm{C}_{2} \varepsilon$ are the closing constants of the RNG $\mathrm{k}-\varepsilon$ model, according to Versteeg and Malalasekera (2007). 


\section{Mesh Generation}

The second stage of the process was the generation of the mesh, which is extremely relevant, because the quality of the mesh is directly linked to the accuracy of the results obtained (Miranda, 2018). Because the modeling was two-dimensional, there were no major difficulties in its generation, only some considerations were made so that the mesh could comprehend the entire area of the domain, as regularly as possible. Figure 3 exemplifies these considerations.
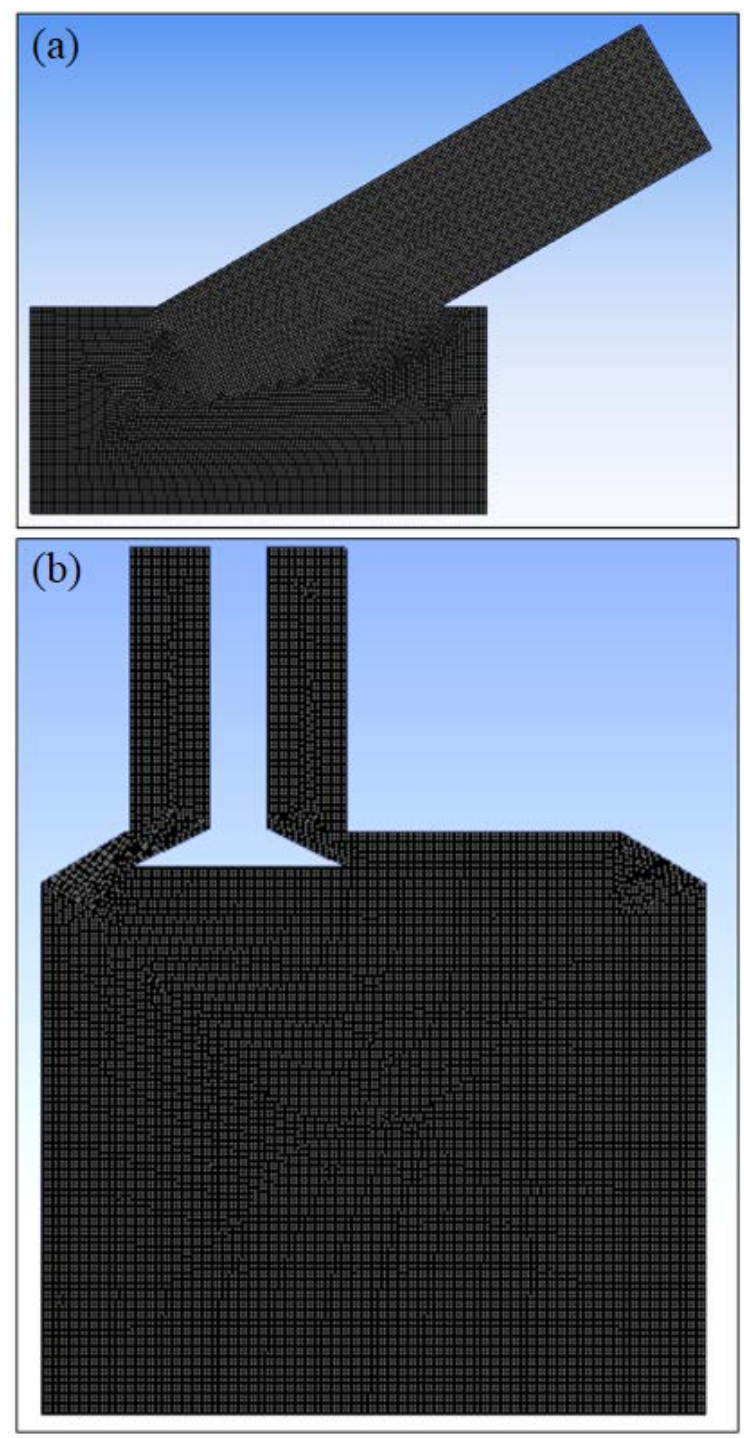

Figure 3. Mesh generation. a) Indirect injection. b) Direct injection.

Because the mesh generated is not fully uniform and has relatively irregular geometry, the discretization method based on finite volumes is the most appropriate to solve the numerical model. The Finite Volume Method divides each cell of the mesh into small volumes, where the calculation is performed using the conservation equations in each of these small volumes, bringing greater precision in the results, so it is the most used method in simulations of CFD, according to Zancanaro (2010).

The mesh generation process, which can be analyzed in Fig. 3, was given through some stages. The first step was the generation of the standard 2D mesh. In this stage it can be observed that the irregularity was higher than expected, as well as its coarse formation.

For a better result, considering some irregularities in the geometry of the domain, the quadrilateral mesh model was chosen for refining, because it better understand its area. As a result, the mesh generation was able to create a number of elements and nodes quite satisfactory, which are respectively, for the indirect injection model 15969 and 15652, and for the direct injection model are 38641 and 38067.

\section{Boundary Conditions}

Once the mesh generation and its refining are finished, the boundary conditions are defined, that is, the physical conditions of analysis.
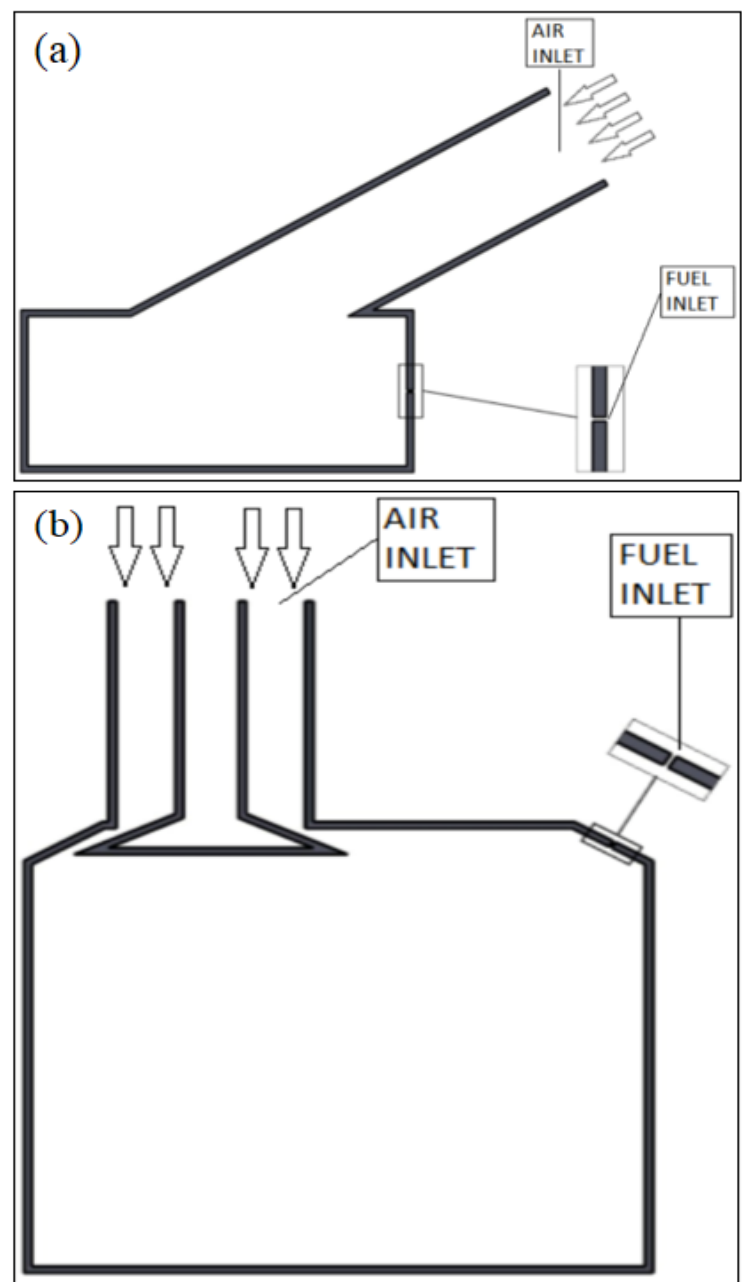

Figure 4. Boundary conditions. a) Indirect injection. b) Direct injection. 
In this step it was defined that the analysis will encompass the air flow at a certain speed and the fuel flow at a certain speed.

Definitions are placed for the entire region of the domain, where the inputs and the walls were specified. Establishing boundary conditions is an indispensable step for the simulation process, because in this stage, the researcher defines and configures the flow paths in its model. Such It can be seen in Fig. 4 that the input definitions have been established, where there is an air inlet and a fuel inlet. All remaining edges were regarded as walls.

The process begins by identifying the walls and naming them. Then the inputs are defined, for this simulation there are only two, which are the air intake and the gasoline inlet. Each of these inputs has specific characteristics of flow velocities, such velocities can be seen in Tab. 1 and Tab. 2 .

Table 1. Air flow speeds and gasoline for indirect injection.

\begin{tabular}{cc}
\hline Air inlet rate $(\mathbf{m} / \mathbf{s})$ & Gas injection rate $(\mathbf{m} / \mathbf{s})$ \\
\hline 23.25 & 28.55 \\
\hline
\end{tabular}

Table 2. Air flow speeds and gasoline for direct injection.

\begin{tabular}{ccc}
\hline Situation & Air inlet rate (m/s) & $\begin{array}{c}\text { Gas injection rate } \\
(\mathbf{m} / \mathbf{s})\end{array}$ \\
\hline I & 23.25 & 90 \\
II & 23.25 & 100 \\
III & 23.25 & 110 \\
\hline
\end{tabular}

Table 1 shows the flow speeds of air intake and gasoline for the simulation model of indirect injection. Such speed represent true flow values in the situation of $3000 \mathrm{rpm}$ with acceleration at $100 \%$. The air intake value was obtained by calculating the flow and displacement volume of the engine, considering the rotation mentioned above.

For gasoline injection speed, the calculation had a greater complexity, since there was not much information available, however, considering the mass of air admitted to the engine cylinder and knowing that the stoichiometric ratio for air-gasoline burning is $15: 1$, it was possible to find the amount of gasoline required to perform burning. Through the analysis of the injection map, it was obtained information that in the condition of $3000 \mathrm{rpm}$ with acceleration at $100 \%$ the injection time is $7 \mathrm{~m} / \mathrm{s}$, and with this its flow rate was found, as well as its speed. Table 2 is related to the flow speed values of air and gasoline for the simulations of direct injection. As the purpose of this work is to compare the efficiency of the mixture homogeneity between indirect injection and direct injection, three situations of direct injection of gasoline were considered, each with different speed so that it was possible to evaluate the improvement in the mixture, taking into consideration the feasibility of the pressure required to inject the gasoline. The amount of gasoline required to burn the intake air to the engine cylinder is the same, both for indirect injection and for direct injection. Considering this, it was concluded that the flow rate is the same for both situations (indirect injection and direct injection), since the stoichiometric ratio remains $15: 1$, so that gasoline can be injected at the speeds described in Tab. 2, alteration in the diameter of orifice and increased pressure were required. These values of speeds and pressures can be observed in Tab. 3 .

Table 3. Relation of speeds and pressures for direct injection.

\begin{tabular}{cc}
\hline Gas injection rate (m/s) & Pressure (bar) \\
\hline 90 & 29.22 \\
100 & 36.06 \\
110 & 43.62 \\
\hline
\end{tabular}

The values shown in Tab. 3 are the result of calculations where the principle of mass continuity was considered, to find the diameter of the orifice, and the Bernoulli's principle to determine the pressure required to inject gasoline at these speeds. These three situations were established by the author, however, they were evaluated taking into consideration the feasibility of manufacturing the components and the plausibility of the results in a realistic context. The need to suggest such situations was due to the particularity of the Univille's Energy Efficiency Project, which the studied engine is part of, and the injector nozzle will be specially dimensioned for this situation. Another factor is the lack of specific information in the literature, regarding the relationship between pressure and injection speed. It is believed that this information is treated confidentially and considered an industrial secret. In order to mix the fuel vapor with the intake air, in such a way that the concentration of fuel and air in any part of the mixture is exactly the same, a study with a target in homogenization is of paramount importance. The characteristic of the mixture has great influence on the combustion process in internal combustion engines, since the flame propagation in the combustion chamber depends on the fuel and the air maintaining a certain proportion in the mixture.

\section{RESULTS AND DISCUSSION}

This chapter will discuss the main results obtained through the simulations. In order to prove this efficiency, this scholarly work focuses on a comparative study of direct injection with indirect injection, through fluid-dynamic simulation, in order to assist in the development of the Univille's Energy efficiency Project from São Bento do Sul. From the standpoint of combustion, the behavior of the mixture does not depend solely on its average composition, 
but mainly on the homogenization of the fuel vapor in the air. Under certain conditions, may have a stoichiometric mixture equal to 1 presenting poor mixing behavior, due to lack of homogenization.

\section{CFD Analysis of Indirect Injection}

Figure 5 shows the results of mass fraction and the streamlines for the indirect injection model, that is, how homogeneous is the mixture and the influence of the velocities in the flows.
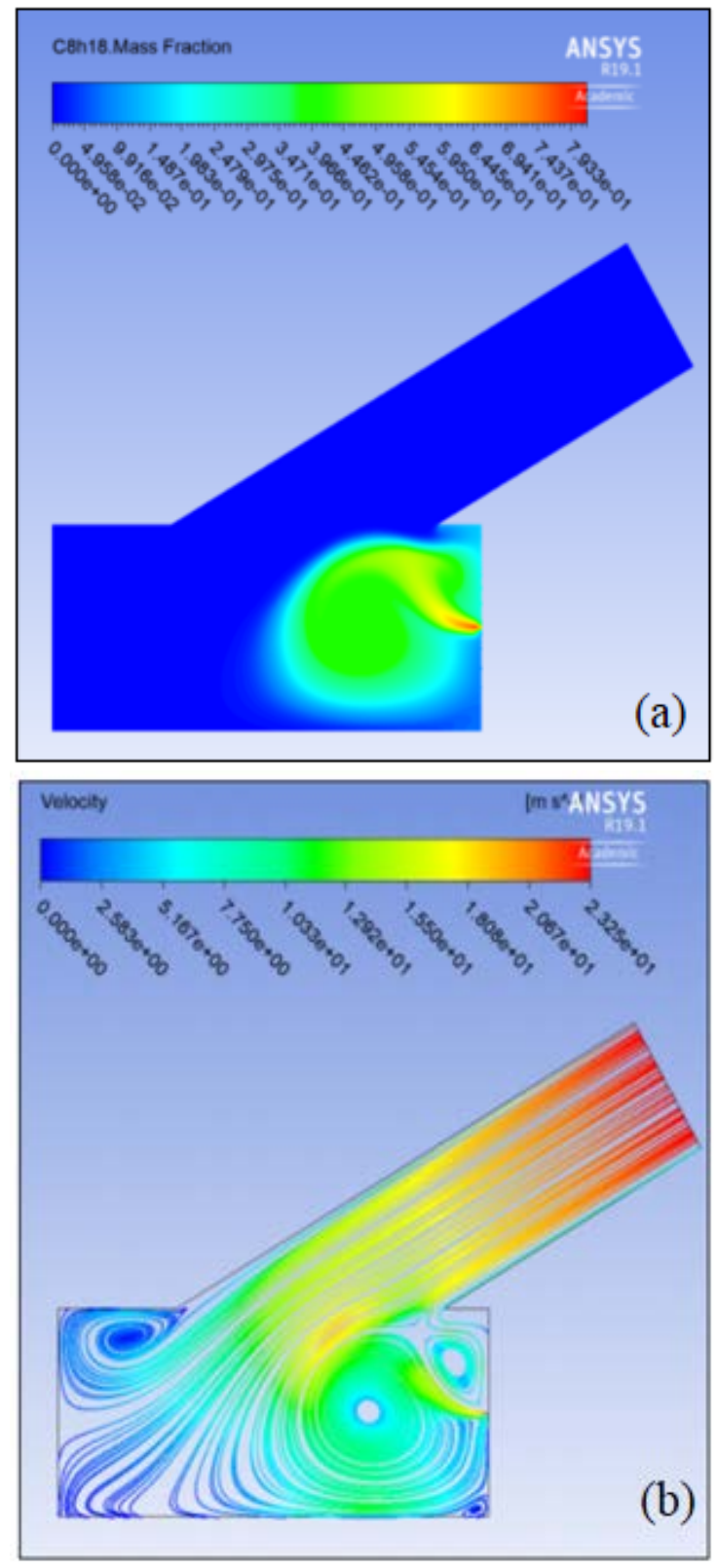

Figure 5. Mixing result. Mass fraction of gasoline in the air. b) Streamlines.

The input velocities are described in Tab. 1. Figure 5 shows that the homogeneity of the mixture is poor, the largest mass fractions of gasoline are present in the region near the injector nozzle, with values ranging from 0.19 to 0.44 . The low quality of the mixture results in inefficient burning, which can be identified as poor mixture, causing greater fuel injection and consequently greater consumption. It can also be observed the representation of the streamlines present in this indirect injection model, where the gasoline injection speeds and the air inlet were exposed in Tab. 1.

The regions of higher speeds are located in the representations of the air intake duct, with a value of $23.25 \mathrm{~m} / \mathrm{s}$ and in a small region that is characterized by the injector nozzle, both with red coloration. It is observed that gasoline injected - with speed as described in Table 1 - suffers reduction of velocity at the moment it is injected, information proven by the graduated ruler, which has a maximum speed of $23.25 \mathrm{~m} / \mathrm{s}$. Throughout its flow, the air begins to lose speed due to the enclosure in the mixing chamber, reaching speeds ranging from 5 to $10 \mathrm{~m} / \mathrm{s}$, which in turn leads to the generation of vortices. The vortices generated in the direction of the injector nozzle have positive effect for the mixture, because they are the ones that through the turbulence caused by the flow, provide the combination of the species, however, the geometry of the mixing chamber does not allow large and efficient vortices, and allied to low gasoline injection speed, the result of the mixture is unsatisfactory.

\section{CFD Analysis of Direct Injection}

The direct injection, as presented throughout this scholarly work, has a gasoline injection system directly in the combustion chamber of the engine, which in turn allows the injection to be performed with higher speed and pressure. This sub-topic will be presented and discussed the results obtained through computerized simulation, three situations of direct injection to the engine geometry were chosen for this study.

The choice of these three situations took into account the feasibility of manufacturing the nozzle and a system with low pressure.

\section{Situation I}

In Fig. 6, it is possible to perceive the first simulation situation, where the flow speeds are described in Tab. 2.

In this situation, represented by Fig. 6, it is already possible to notice an expressive improvement in the quality of the mixture, compared to the indirect injection model. The mass fraction of gasoline in the air has a higher concentration region in the lower right corner of the combustion chamber, characterized by a green color with values ranging from 0.41 to 0.46 . Due to the behavior of the air flow, along with the speed of gasoline injection - described in Tab. 2 - It is noted that there are several points where the mass fraction reduces, towards the center of the chamber for example, ranging from 0.2 to 0.3 . 
The mass fraction values decrease along the flow, in the tangent regions to the center of the chamber, on the right side reaches values from 0.15 to 0.2 , and decreases further on the left side, with values ranging from 0.05 to 0.1 .
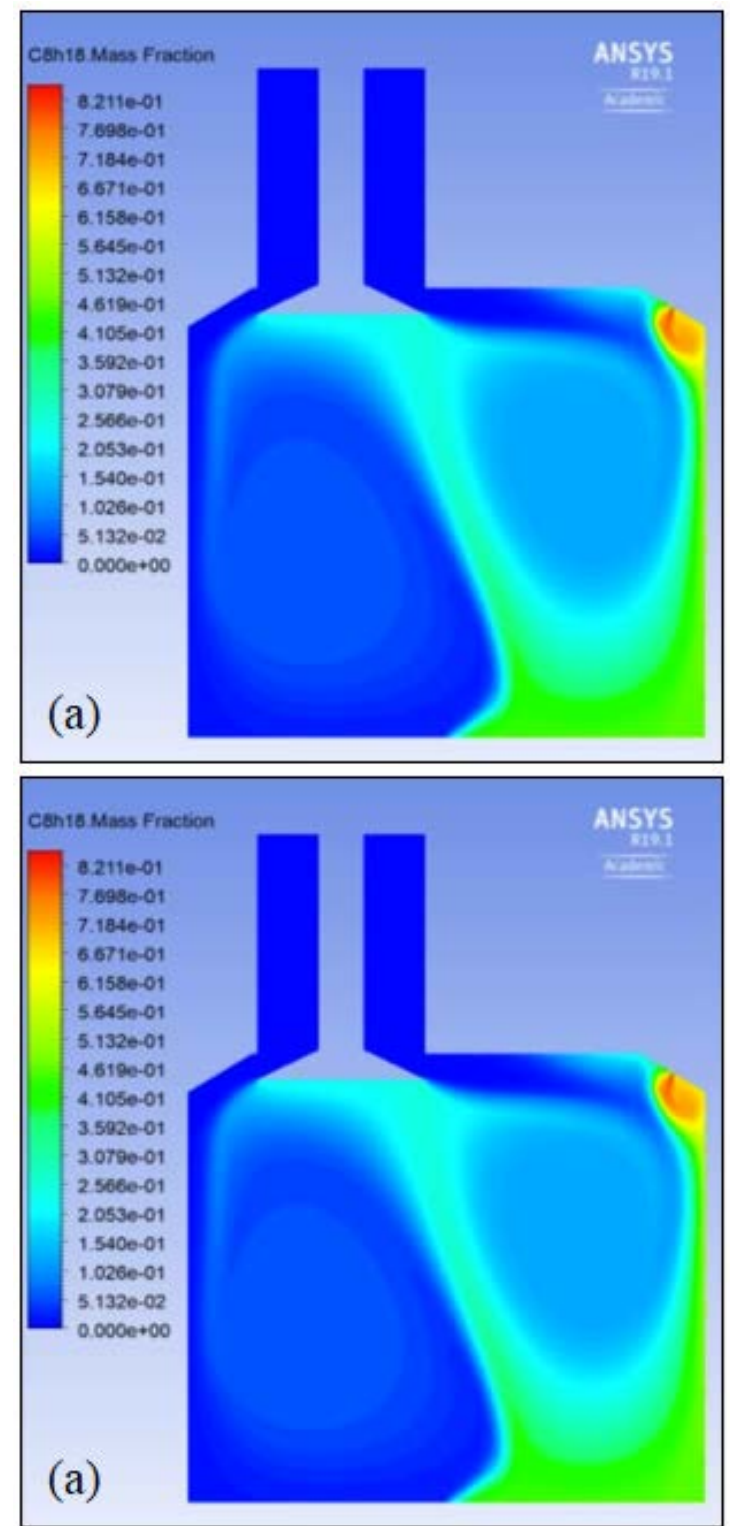

Figure 6. Situation I. a) Mass fraction of gasoline in the air. b) Streamlines.

This means that the turbulence caused by the air flow provided a relatively good homogeneity.

Another important factor to be considered is the speed of gasoline injection, which by the fact of being higher, provided greater air penetration. It is observed the increase of the air flow speed when it passes through the inlet valve, an increase justified by the reduction of area in this section and represented by the red color in the image, reaching the maximum value of $67.94 \mathrm{~m} / \mathrm{s}$.

The turbulence caused by this flow generates two large vortices, where each one occupies practically half of the combustion chamber with speeds from 7.5 to $22.6 \mathrm{~m} / \mathrm{s}$. The air flow in this geometry makes it possible to carry the gasoline in a way more efficient, where this is demonstrated in the upper right corner of the combustion chamber, in which the speed of gasoline is reduced by contact with air turbulence, equating the flow rate of the same, ranging from 30 to $45.2 \mathrm{~m} / \mathrm{s}$.

\section{Situation II}

In this situation, a higher speed of gasoline injection was considered in the simulation, as shown in Tab. 2. Figure 7a shows the result corresponding to the mass fraction of this situation and the flow Fig. 7b shows that the concentration of gasoline inside the combustion chamber behaves similar to Situation I.
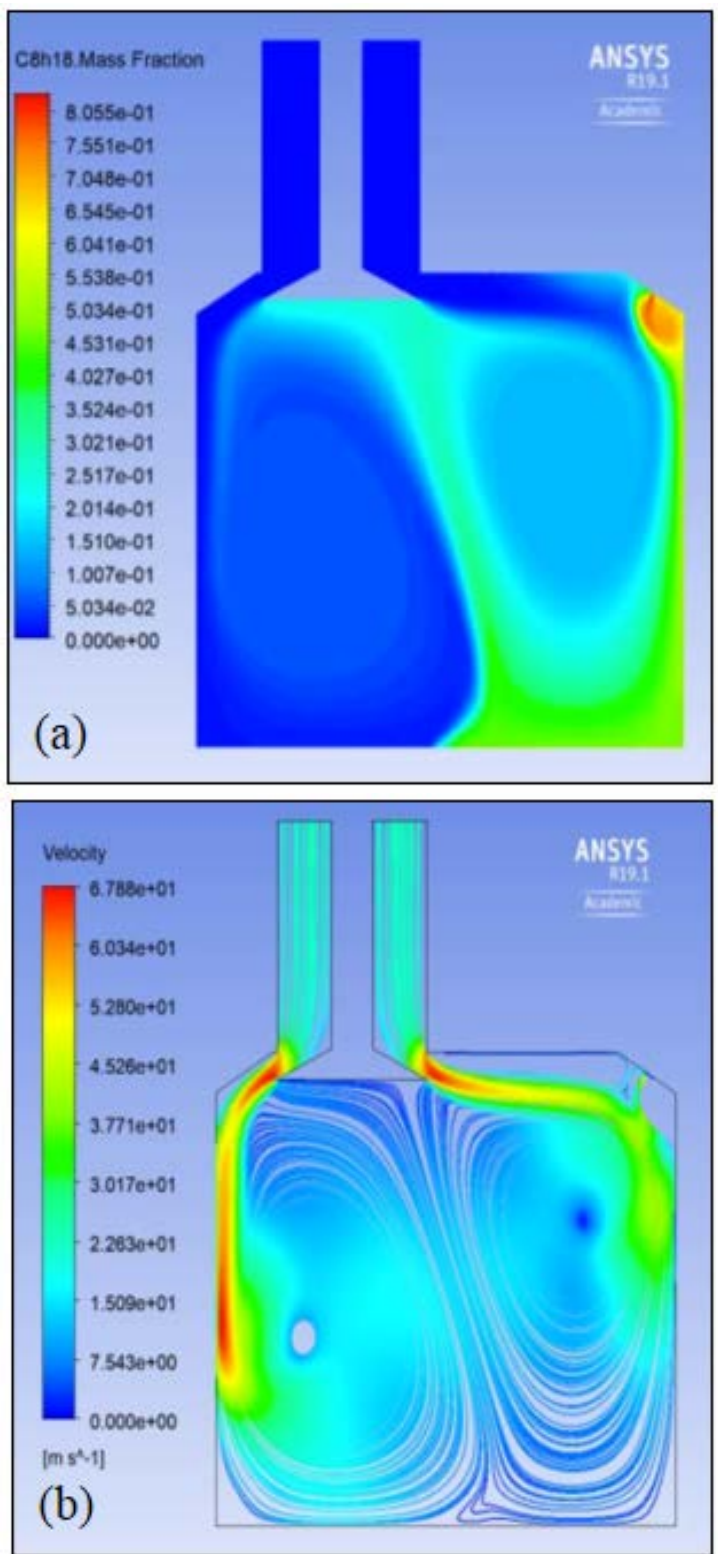

Figure 7. Situation II. a) Mass fraction of gasoline in the air. b) Streamlines. 
The mass fraction of gasoline in the air still has a higher concentration region in the lower right corner - represented by the green color and with values varying from 0.37 to 0.47 - and decreases towards the center of the combustion chamber to the bottom of the inlet valve, dividing the combustion chamber into two, region presenting values from 0.2 to 0.35 for mass fraction and $15 \mathrm{~m} / \mathrm{s}$ for speed. In the right side region, there is a large vortex, which was responsible for the conduction of gasoline, moving at a speed of approximately $30 \mathrm{~m} / \mathrm{s}$ and gradually decreasing to approximately $7.5 \mathrm{~m} / \mathrm{s}$.

It is also possible to realize that, in this great vortex the mixture of the species presented a better result, denoting values between 0.15 and 0.25 . Gasoline travels the vortex-oriented path until it reaches the other large vortex, which is located in the region on the left side of the combustion chamber. This other vortex participates in the generation of the mixture by shifting a small mass fraction of gasoline to the region on the left side, which exposes values between 0.05 and 0.1 .

\section{Situation III}

In situation III, the simulation obtained the same essence, only with change in the speed of fuel injection, as shown in Tab. 2. The results are shown in Fig. 8.

It is noted in Fig. 8, in the case of a mass fraction, that the region with the highest concentration of gasoline continues in the lower right corner of the combustion chamber, in this case it is perceived that there was an increase in this concentration, ranging the mass fraction from 0.40 to 0.55 .

The concentration decreased in the direction of the center of the chamber, presenting values from 0.2 to 0.37 , and it is divided by two large vortices generated by the air flow, where the speeds start at $67.83 \mathrm{~m} / \mathrm{s}$ - region of the inlet valve - and reduce over the vortices to $7.5 \mathrm{~m} / \mathrm{s}$.

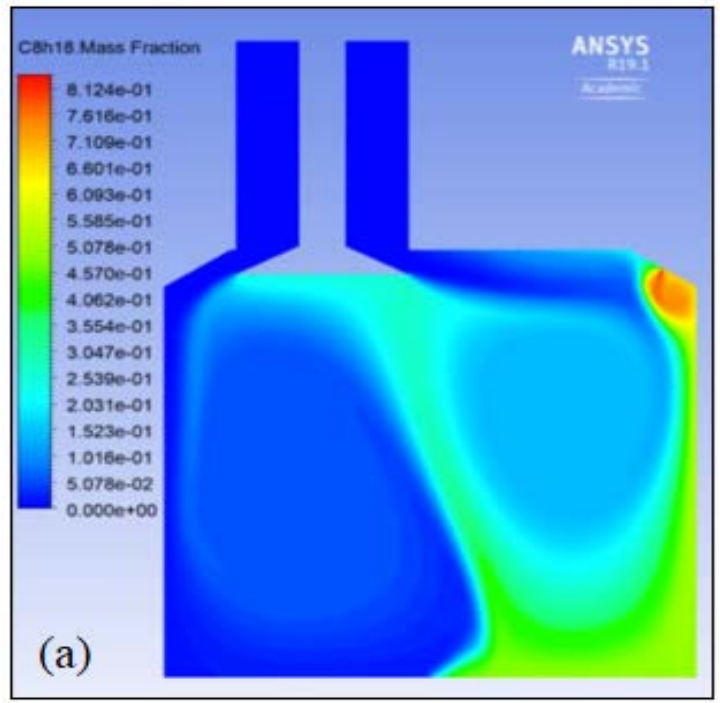

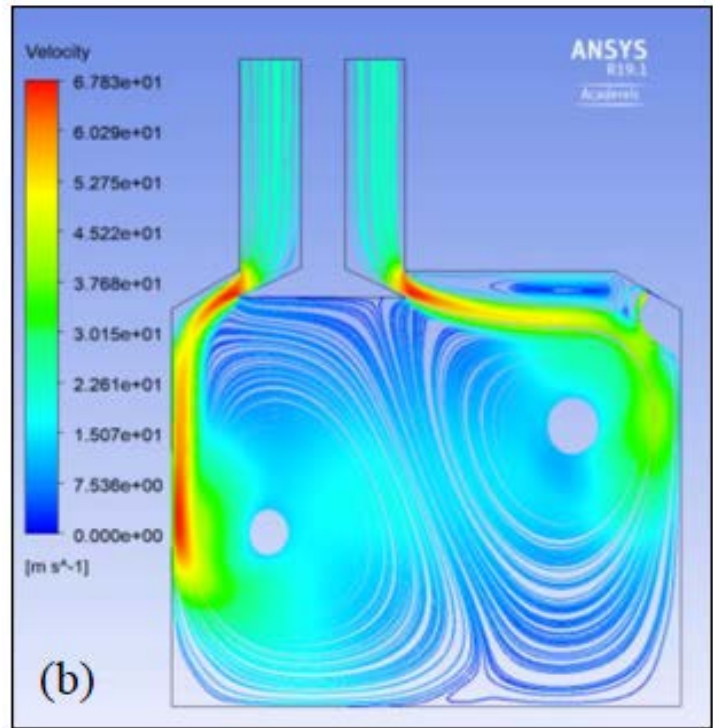

Figure 8. Situation III. a) Mass fraction of gasoline in the air. b) Streamlines.

In this division can be perceived, on the right side, better quality regions in the mixture, exhibiting values of mass fraction between 0.2 and 0.3 - for the right side - and values between 0.05 to 0.1 for the left side.

The speed of gasoline injection manifested the same behavior in terms of deceleration. At the instant of the injection, gasoline promptly initiated the penetration into the turbulent air and equaled its velocity at air velocity, assuming values at the beginning of its flow between 37 to $52 \mathrm{~m} / \mathrm{s}$.

\section{CONCLUSIONS}

In order to compare the efficiency in the homogeneity of the air-fuel mixture in the UMK 435T engine used for the Energy Efficiency Program of Univille - Campus São Bento do Sul, four simulations were made. The result of the simulation of the indirect injection was obtained and subsequently compared to the results obtained with the three direct injection situations. With the results achieved, it can be concluded that the evidences exposed in the images demonstrating the mass fractions and streamlines prove superior efficiency in the quality of the air-fuel mixture in the direct injection systems.

It can also be concluded that the air displacement has a great influence on the homogeneity of the mixture, an influence shared equally at the rate of gasoline injection. Although the results showed that gasoline assumed the speed of air flow at the same instant it was injected, the fact of being injected with high speed provided good air penetration, and these are two factors of extreme relevance to development and design of engines, provided they are balanced, as shown in Situation III, where the speed of gasoline injection was higher, for 
the same behavior of air intake, the results showed lower homogeneity when compared to Situation I.

Thus, it is concluded that among all simulated situations, both for the indirect injection model and for the direct injection model, the Situation I, where the gasoline injection speed is $90 \mathrm{~m} / \mathrm{s}$, presents the best results of air-fuel mixture formation, taking into consideration the feasibility of manufacturing for a future project execution, since it does not require great pressures to provide this speed.

\section{REFERENCES}

Guzzo, M. E., 2012, Metodologias para Análise e Caracterização dos Sprays de um Injetor de Injeção Direta de Gasolina, Master Thesis, PPGMEC-UFMG, Belo Horizonte, MG. (in Portuguese).

Kasper, F. R. S., 2003, Modelagem e Simulação de Motores à Combustão Interna por Técnicas da Fluidodinâmica Computacional (CFD), Master Thesis, UNICAMP, Campinas, SP. (in Portuguese).

Kim, S. J., Kim, Y. N., and Lee, J. H., 2008, Analysis of the in-Cylinder Flow, Mixture Formation and Combustion Processes in a Spray-Guided GDI Engine, SAE Technical Paper Series.

Kim, Y. J., Lee, S. H., and Cho, N. H., 1999, Effect of Air Motion on Fuel Spray Characteristics in a Gasoline Direct Injection Engine, SAE Technical Paper Series.

Milton, B. E., Behnia, M., and Ellerman, D. M., 2001, Fuel Deposition and Re-Atomosation Fuel/Air Flows through Engine Inlet Valves, International Jornal of Heat and Fluid Flow, Vol. 22, pp. 350-357.

Miranda, D. A., Cristofolini, R., Corazza, E. J., Santos, G. J., and Amaral, C. E., 2018, Comparison of Mathematical Methods to Obtain Concentration and Temperature of Newtonian Fluids in Tubular Reactors, Open Access Library Journal , Vol. 5, pp. $1-8$.

Miranda, D. A., 2018, Influence of Mesh Geometry and Mesh Refinement on Mathematical Models of Thermoplastic Injection Simulation Tools, IOSR Journal of Mechanical and Civil Engineering, Vol. 15, pp. 38-44.

Schreiner, A., 2014, Injeção Eletrônica para Eficiência Energética, CET - Ciências Exatas e Tecnológicas. (in Portuguese).

Versteeg, H. K., and Malalasekera, W., 2007, An Introduction to Computational Fluid Dynamics: The Finite Volume Method, 2nd Edition, Harlow.

Zancanaro, F. V. J., 2010, Simulação Numérica do Escoamento Turbulento em Motores de Combustão Interna, Master Thesis, PROMECUFRGS, Porto Alegre, RG. (in Portuguese). 